\title{
Local Defect Correction for Time-Dependent Partial Differential Equations
}

\author{
Remo Minero, Martijn J.H. Anthonissen, and Robert M.M. Mattheij \\ Eindhoven University of Technology, Department of Mathematics and Computer \\ Science, Den Dolech 2, P.O. Box 513, 5600MB Eindhoven, the Netherlands. \\ $\{r$.minero,m.j.h.anthonissen,r.m.m.mattheij\}@tue.nl
}

A Local Defect Correction (LDC) method for solving time-dependent partial differential equations whose solutions have highly localized properties is discussed. We present some properties of the technique. Results of numerical experiments illustrate the accuracy and the efficiency of the method.

\section{Introduction}

Solutions of Partial Differential Equations (PDEs) are often characterized by highly localized properties. Examples are frequently encountered in the area of shock hydrodynamics, transport in turbulent flow fields, combustion, etc. An efficient solution of this kind of problems requires the usage of adaptive grid techniques, where a fine grid spacing and, possibly, a small time step are adopted only where the high activity occurs. Among other techniques, the Local Defect Correction (LDC) method for time-dependent problems described in [MAM04] has the advantage that only uniform grid and uniform grid solvers can be used. At each time step, LDC is an iterative process in which a global coarse grid solution and a local fine grid solution are iteratively improved. In particular, the local approximation improves the solution globally through a defect correction.

The LDC method was introduced in [Hac84] for solving elliptic boundary value problems. $\mathrm{LDC}$ is a domain decomposition technique in which the local domain fully overlaps the global one. An analysis of LDC in combination with finite differences is presented in [FR96]. In [Ant05] the method is extended to include adaptivity, multilevel refinement, domain decomposition and regridding. In this paper we present the LDC technique for solving time-dependent PDEs (Section 2) and we discuss some properties of the method (Section 3). Results of numerical experiments illustrate the accuracy and the efficiency of the method (Section 4). 


\section{The LDC method}

We consider the following two-dimensional problem

$$
\begin{cases}\frac{\partial u(\mathbf{x}, t)}{\partial t}=L u(\mathbf{x}, t)+f(\mathbf{x}, t), & \text { in } \Omega \times \Theta, \\ u(\mathbf{x}, t)=\psi(\mathbf{x}, t), & \text { on } \partial \Omega \times \Theta, \\ u(\mathbf{x}, 0)=\varphi_{0}(\mathbf{x}), & \text { in } \Omega \cup \partial \Omega,\end{cases}
$$

where $\Omega$ is a spatial domain, $\partial \Omega$ its boundary and $\Theta$ the time interval $\left(0, t_{\text {end }}\right]$. Moreover, $L$ is a linear differential operator, $f$ a source term, $\psi$ a Dirichlet boundary condition and $\varphi_{0}$ a given initial condition.

Problem (1) has to be discretized in space and time in order to be solved numerically. For this reason, we introduce a global uniform coarse grid (grid size $H$ ), which we denote by $\Omega^{H}$. We also introduce the time step $\Delta t$. We assume that $u$ has, at each time level, a region of high activity that covers a small part of $\Omega$. At time $t_{n}:=n \Delta t$ a coarse grid approximation computed with a time step $\Delta t$ might be not adequate enough to represent $u\left(\mathbf{x}, t_{n}\right)$. In order to better capture the local high activity, we introduce a local uniform fine grid (grid size $h<H$ ), which we denote by $\Omega_{l}^{h}$. On $\Omega_{l}^{h}$ the time integration is performed using a time step $\delta t=\Delta t / \tau$, with $\tau$ an integer $\geq 1$. In LDC the local solution is used to improve the global approximation through a defect correction.

In the remainder of this section we will assume that a solution $u^{H, h, n-1}$ is known at time $t_{n-1}$ on the composite grid $\Omega^{H, h}:=\Omega^{H} \cup \Omega_{l}^{h}$, see Fig. 1. Its expression is given by

$$
u^{H, h, n-1}:= \begin{cases}u_{l}^{h, n-1}, & \text { in } \Omega_{l}^{h}, \\ u^{H, n-1}, & \text { in } \Omega^{H} \backslash \Omega_{l}^{h},\end{cases}
$$

where $u_{l}^{h, n-1}$ and $u^{H, n-1}$ are a local and a global approximation of $u\left(\mathbf{x}, t_{n-1}\right)$ respectively. We want to compute an approximation of the solution at the new time level $t_{n}$ on the composite grid.

\section{Coarse grid problem}

A first coarse grid approximation at $t_{n}$, we call it $u_{0}^{H, n}$, can be computed applying the backward Euler method to the PDE in (1). The usage of explicit time integrators on the global grid is not of interest in LDC; this is discussed in [MAM04]. We obtain

$$
\left(I-\Delta t L^{H}\right) u_{0}^{H, n}=\left.u^{H, h, n-1}\right|_{\Omega^{H}}+f^{H, n} \Delta t,
$$

where $L^{H}$ is some spatial discretization of $L$. In (3), $f^{H, n}$ also includes the Dirichlet boundary conditions. We rewrite (3) as 


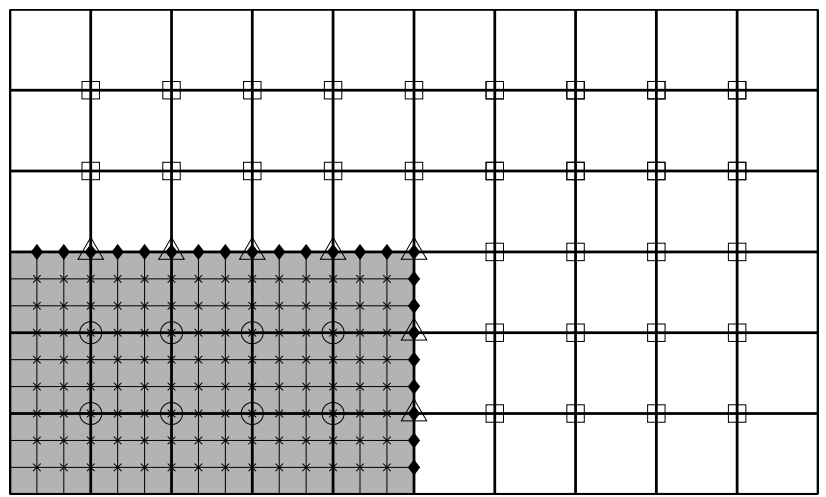

Fig. 1. Example of composite grid $\Omega^{H, h}$

$$
M^{H} u_{0}^{H, n}=\left.u^{H, h, n-1}\right|_{\Omega^{H}}+f^{H, n} \Delta t .
$$

We assume $M^{H}$ to be invertible. We denote by $\Gamma$ the interface between $\Omega_{l}$ and $\Omega \backslash \Omega_{l}$. For convenience we partition the coarse grid points as follows

$$
\Omega^{H}=\Omega_{l}^{H} \cup \Gamma^{H} \cup \Omega_{c}^{H},
$$

where $\Omega_{l}^{H}:=\Omega^{H} \cap \Omega_{l}, \Gamma^{H}:=\Omega^{H} \cap \Gamma$ and $\Omega_{c}^{H}:=\Omega^{H} \backslash\left(\Omega_{l}^{H} \cup \Gamma^{H}\right)$. In Fig. 1 the coarse grid points $\Omega_{l}^{H}$ are marked with circles, while the points $\Gamma^{H}$ and $\Omega_{c}^{H}$ are denoted by triangles and squares respectively. Assuming that the spatial discretization on the coarse grid is such that the stencil at grid point $(x, y)$ involves at most function values at $(x+i H, y+j H)$, with $i, j \in\{-1,0,1\}$, we can rewrite (4) as

$$
\left(\begin{array}{ccc}
M_{l}^{H} & B_{l, \Gamma}^{H} & 0 \\
B_{\Gamma, l}^{H} & M_{\Gamma}^{H} & B_{\Gamma, c}^{H} \\
0 & B_{c, \Gamma}^{H} & M_{c}^{H}
\end{array}\right)\left(\begin{array}{c}
u_{l, 0}^{H, n} \\
u_{\Gamma, 0}^{H, n} \\
u_{c, 0}^{H, n}
\end{array}\right)=\left(\begin{array}{l}
\left.u^{H, h, n-1}\right|_{\Omega_{l}^{H}} \\
\left.u^{H, h, n-1}\right|_{\Gamma^{H}} \\
\left.u^{H, h, n-1}\right|_{\Omega_{c}^{H}}
\end{array}\right)+\left(\begin{array}{l}
f_{l}^{H, n} \Delta t \\
f_{\Gamma}^{H, n} \Delta t \\
f_{c}^{H, n} \Delta t
\end{array}\right) .
$$

\section{Fine grid problem}

In order to formulate a discrete problem on $\Omega_{l}^{h}$, we have to define artificial boundary conditions on $\Gamma$. We can prescribe artificial Dirichlet boundary conditions at time $t_{n}$ by applying an interpolation operator in space $P^{h, H}$. The operator $P^{h, H}$ maps function values in $\Gamma^{H}$ to function values at grid points of the fine grid that lie on the interface, denoted by $\Gamma^{h}$. In Fig. 1 the points $\Gamma^{h}$ are marked with small diamonds. If we want to perform time integration with a time step $\delta t=\Delta t / \tau$, we also need to provide boundary conditions on $\Gamma^{h}$ at all the intermediate time levels $t_{n-1+k / \tau}$, with $k=1,2, \ldots, \tau-1$. Therefore we perform linear time interpolation between $\left.u^{H, h, n-1}\right|_{\Gamma^{h}}$ and $P^{h, H} u_{0}^{H, n}$. A fine grid approximation at time $t_{n}$ can thus be computed solving 


$$
\begin{aligned}
& M_{l}^{h} u_{l, 0}^{h, n-1+k / \tau}=u_{l, 0}^{h, n-1+(k-1) / \tau}+f_{l}^{h, n-1+k / \tau} \delta t \\
& \quad-B_{l, \Gamma}^{h}\left(\frac{k}{\tau} P^{h, H} u_{\Gamma, 0}^{H, n}+\left.\frac{\tau-k}{\tau} u^{H, h, n-1}\right|_{\Gamma^{h}}\right), \quad \text { for } k=1,2, \ldots, \tau .
\end{aligned}
$$

The procedure (7) is initialized using

$$
u_{l, 0}^{h, n-1}=\left.u^{H, h, n-1}\right|_{\Omega_{l}^{h}}
$$

We can combine all the equations in (7) to express $u_{l, w}^{h, n}$, with $w=0$, directly in terms of $\left.u^{H, h, n-1}\right|_{\Omega_{l}^{h}}$. We obtain

$$
\begin{aligned}
\left(M_{l}^{h}\right)^{\tau} u_{l, w}^{h, n} & =\left.u^{H, h, n-1}\right|_{\Omega_{l}^{h}}+\sum_{k=1}^{\tau}\left(M_{l}^{h}\right)^{k-1} f_{l}^{h, n-1+k / \tau} \delta t \\
& -\sum_{k=1}^{\tau}\left(M_{l}^{h}\right)^{k-1} B_{l, \Gamma}^{h}\left(\frac{k}{\tau} P^{h, H} u_{\Gamma, w}^{H, n}+\left.\frac{\tau-k}{\tau} u^{H, h, n-1}\right|_{\Gamma^{h}}\right),
\end{aligned}
$$

or

$$
\left(M_{l}^{h}\right)^{\tau} u_{l, w}^{h, n}=\left.u^{H, h, n-1}\right|_{\Omega_{l}^{h}}+F_{l}^{h, n} \delta t-W_{l, \Gamma}^{n} P^{h, H} u_{\Gamma, w}^{H, n}+\left.Z_{l, \Gamma}^{n} u^{H, h, n-1}\right|_{\Gamma^{h}} .
$$

In (10) $F_{l}^{h, n}$ depends only on the source term and on the fine grid operator $M_{l}^{h}$, while $W_{l, \Gamma}^{n}$ and $Z_{l, \Gamma}^{n}$ only depend on $M_{l}^{h}$ and $B_{l, \Gamma}^{h}$.

\section{Defect correction and LDC iteration}

The fine grid approximation is now used to overall improve the coarse grid solution at $t_{n}$. The fine grid solution is regarded to be more accurate than the coarse grid approximation because it is computed with a grid size $h<H$ and a time step $\delta t \leq \Delta t$. The fine grid solution can therefore be used to approximate the local discretization error or defect in $\Omega_{l}^{H}$. For $w=1$, the approximated defect is given by (cf. the first equation in (6))

$$
\tilde{d}_{l, w-1}^{H, n}:=M_{l}^{H} R^{H, h} u_{l, w-1}^{h, n}+B_{l, \Gamma}^{H} u_{\Gamma, w-1}^{H, n}-\left.u^{H, h, n-1}\right|_{\Omega_{l}^{H}}-f_{l}^{H, n} \Delta t,
$$

where $R^{H, h}$ is a restriction operator from the fine to the coarse grid, such that $\left(R^{H, h} u_{l, w-1}^{h, n}\right)(x, y)=u_{l, w-1}^{h, n}(x, y)$ for every $(x, y) \in \Omega_{l}^{H}$. The defect $\tilde{d}_{l, w-1}^{H, n}$ is now added to the right hand side of (6). A more accurate coarse grid approximation can be computed solving

$$
\begin{aligned}
M^{H} u_{w}^{H, n} & =\left(\begin{array}{l}
\left.u^{H, h, n-1}\right|_{\Omega_{l}^{H}} \\
\left.u^{H, h, n-1}\right|_{\Gamma^{H}} \\
\left.u^{H, h, n-1}\right|_{\Omega_{c}^{H}}
\end{array}\right)+\left(\begin{array}{c}
f_{l}^{H, n} \Delta t+\tilde{d}_{l, w-1}^{H, n} \\
f_{\Gamma}^{H, n} \Delta t \\
f_{c}^{H, n} \Delta t
\end{array}\right) \\
& =\left(\begin{array}{c}
0 \\
\left.u^{H, h, n-1}\right|_{\Gamma^{H}} \\
\left.u^{H, h, n-1}\right|_{\Omega_{c}^{H}}
\end{array}\right)+\left(\begin{array}{c}
M_{l}^{H} R^{H, h} u_{l, w-1}^{h, n}+B_{l, \Gamma}^{H} u_{\Gamma, w-1}^{H, n} \\
f_{\Gamma}^{H, n} \Delta t \\
f_{c}^{H, n} \Delta t
\end{array}\right) .
\end{aligned}
$$


The new coarse grid solution can be used to update the boundary conditions for a new local problem on $\Omega_{l}^{h}$, which in turn will correct the coarse grid approximation. At each time step the LDC method is thus an iterative procedure and, as studied in [AMTB03] for stationary cases, its convergence is very fast.

\section{Adaptivity}

In a time dependent problem it is likely that the high activity moves as time proceeds. As a consequence, the local region $\Omega_{l}$ might be located in different positions and a have a different size or shape at the various time levels in $\Theta$. At each time, in order to perform the next time step $\Delta t$, we have to determine a suitable $\Omega_{l}$. This can be done, for example, by measuring some characteristics of the solution (e.g. slope, gradients, etc.). Many methods have proposed in the literature, see for example [BS99]. If the composite grid changes in time, we interpolate the solution found at $t_{n-1}$ to the new grid to construct the initial solution $u^{H, h, n-1}$.

\section{Properties of the LDC method}

In this section we will discuss some properties of the LDC method for timedependent PDEs. The following lemma shows that once the coarse grid approximations do not change on the interface $\Gamma$, the LDC algorithm converges and a fixed point of the iteration has been reached.

Lemma 1. If $u_{\Gamma, w}^{H, n}=u_{\Gamma, w-1}^{H, n}$ for a certain index $w$, then the LDC iteration converges and

$$
u_{q}^{H, n}=u_{w}^{H, n}, \quad u_{q}^{h, n}=u_{w}^{h, n},
$$

for all $q=w, w+1, \ldots$

Proof. Assume that $u_{\Gamma, w}^{H, n}=u_{\Gamma, w-1}^{H, n}$ for a certain index $w$. From (10), we have that $u_{w}^{h, n}=u_{w-1}^{h, n}$, and hence, from (12),

$$
\begin{aligned}
M^{H} u_{w+1}^{H, n} & =\left(\begin{array}{c}
0 \\
\left.u^{H, h, n-1}\right|_{\Gamma^{H}} \\
\left.u^{H, h, n-1}\right|_{\Omega_{c}^{H}}
\end{array}\right)+\left(\begin{array}{c}
M_{l}^{H} R^{H, h} u_{l, w}^{h, n}+B_{l, \Gamma}^{H} u_{\Gamma, w}^{H, n} \\
f_{\Gamma}^{H, n} \Delta t \\
f_{c}^{H, n} \Delta t
\end{array}\right) \\
& =\left(\begin{array}{c}
0 \\
\left.u^{H, h, n-1}\right|_{\Gamma^{H}} \\
\left.u^{H, h, n-1}\right|_{\Omega_{c}^{H}} ^{H,}
\end{array}\right)+\left(\begin{array}{c}
M_{l}^{H} R^{H, h} u_{l, w-1}^{h, n}+B_{l, \Gamma}^{H} u_{\Gamma, w-1}^{H, n} \\
f_{\Gamma}^{H, n} \Delta t \\
f_{c}^{H, n} \Delta t
\end{array}\right)=M^{H} u_{w}^{H, n}
\end{aligned}
$$

Because we have assumed $M^{H}$ to be invertible, we have $u_{w+1}^{H, n}=u_{w}^{H, n}$, for all grid points in $\Omega_{H}$. Since $\Gamma_{H} \subset \Omega_{H}$, we have $u_{\Gamma, w+1}^{H, n}=u_{\Gamma, w}^{H, n}$. By induction, we find $u_{q}^{H, n}=u_{w}^{H, n}$ and $u_{q}^{h, n}=u_{w}^{h, n}$, for all $q=w, w+1, \ldots$ 
We can combine (12), (11) and (10), and express the LDC iteration as

$$
\begin{gathered}
\left(\begin{array}{cccc}
\left(M_{l}^{h}\right)^{\tau} & 0 & W_{l, \Gamma}^{n} P^{h, H} & 0 \\
0 & M_{l}^{H} & B_{l, \Gamma}^{H} & 0 \\
0 & B_{\Gamma, l}^{H} & M_{\Gamma}^{H} & B_{\Gamma, c}^{H} \\
0 & 0 & B_{c, \Gamma}^{H} & M_{c}^{H}
\end{array}\right)\left(\begin{array}{c}
u_{l, w}^{h, n} \\
u_{l, w}^{H, n} \\
u_{\Gamma, w}^{H, n} \\
u_{c, w}^{H, n}
\end{array}\right)=\left(\begin{array}{cccc}
0 & 0 & 0 & 0 \\
M_{l}^{H} R^{H, h} & 0 & B_{l, \Gamma}^{H} & 0 \\
0 & 0 & 0 & 0 \\
0 & 0 & 0 & 0
\end{array}\right)\left(\begin{array}{c}
u_{l, w-1}^{h, n} \\
u_{l, w-1}^{H, n} \\
u_{\Gamma, w-1}^{H, n} \\
u_{c, w-1}^{H, n}
\end{array}\right) \\
+\left(\begin{array}{c}
\left.u^{H, h, n-1}\right|_{\Omega_{l}^{h}} \\
0 \\
\left.u^{H, h, n-1}\right|_{\Gamma_{H}} \\
\left.u^{H, h, n-1}\right|_{\Omega_{c}^{H}} ^{H, n}
\end{array}\right)+\left(\begin{array}{c}
F_{l}^{h, n} \Delta t \\
0 \\
f_{\Gamma}^{H, n} \delta t \\
f_{c}^{H, n} \delta t
\end{array}\right)+\left(\begin{array}{c}
\left.Z_{l, \Gamma}^{n} u^{H, h, n-1}\right|_{\Gamma_{H}} \\
0 \\
0 \\
0
\end{array}\right) .
\end{gathered}
$$

We rewrite (14) using the short notation

$$
M^{H, h} u_{w}^{H, h, n}=S^{H, h} u_{w-1}^{H, h, n}+\tilde{u}^{H, h, n-1}+\tilde{f}^{H, h, n}+\tilde{z}^{H, h, n-1} .
$$

If the LDC algorithm converges, then (15) has a fixed point, which we denote by $u^{H, h, n}$ (we remove the subscript that numbers the LDC iterations). The fixed point $u^{H, h, n}$ satisfies by definition

$$
M^{H, h} u^{H, h, n}=S^{H, h} u^{H, h, n}+\tilde{u}^{H, h, n-1}+\tilde{f}^{H, h, n}+\tilde{z}^{H, h, n-1} .
$$

The following theorem states that, if the LDC iteration converges, the fine and the coarse grid approximation coincide in the common points between fine and coarse grid.

Theorem 1. Assume that the LDC iteration converges. Then $u^{H, h, n}$ is such that the projection of $u_{l}^{h, n}$ on the local coarse grid equals $u_{l}^{H, n}$, viz.

$$
R^{H, h} u_{l}^{h, n}=u_{l}^{H}
$$

Proof. Combination of (16) and (14) yields

$$
\left(\begin{array}{cccc}
\left(M_{l}^{h}\right)^{\tau} & 0 & W_{l, \Gamma}^{n} P^{h, H} & 0 \\
-M_{l}^{H} R^{H, h} & M_{l}^{H} & 0 & 0 \\
0 & B_{\Gamma, l}^{H} & M_{\Gamma}^{H} & B_{\Gamma, c}^{H} \\
0 & 0 & B_{c, \Gamma}^{H} & M_{c}^{H}
\end{array}\right)\left(\begin{array}{c}
u_{l}^{h, n} \\
u_{l}^{H, n} \\
u_{\Gamma}^{H, n} \\
u_{c}^{H, n}
\end{array}\right)=\tilde{u}^{H, h, n-1}+\tilde{f}^{H, h, n}+\tilde{z}^{H, h, n-1} .
$$

The second equation of the system reads

$$
M_{l}^{H} R^{H, h} u_{l}^{h, n}+M_{l}^{H} u_{l}^{H, n}=0,
$$

which gives (17), since we supposed $M^{H}$ (and hence $M_{l}^{H}$ ) to be invertible. 
We finally write the system of equations that the limit of the LDC iteration satisfies at time $t_{n}$.

Theorem 2. Assume that the LDC iteration converges. Then $u_{l}^{h, n}, u_{\Gamma}^{H, n}$ and $u_{c}^{H, n}$ satisfy the following system of equations

$$
\begin{gathered}
\left(\begin{array}{ccc}
\left(M_{l}^{h}\right)^{\tau} & W_{l, \Gamma}^{n} P^{h, H} & 0 \\
B_{\Gamma, l}^{H} R^{H, h} & M_{\Gamma}^{H} & B_{\Gamma, c}^{H} \\
0 & B_{c, \Gamma}^{H} & M_{c}^{H}
\end{array}\right)\left(\begin{array}{c}
u_{l, w}^{h, n} \\
u_{\Gamma, w}^{H, n} \\
u_{c, w}^{H, n}
\end{array}\right) \\
\quad=\left(\begin{array}{l}
\left.u^{H, h, n-1}\right|_{\Omega_{l}^{h}} \\
\left.u^{H, h, n-1}\right|_{\Gamma_{H}} \\
\left.u^{H, h, n-1}\right|_{\Omega_{c}^{H}} ^{H}
\end{array}\right)+\left(\begin{array}{c}
F_{l}^{h, n} \Delta t \\
f_{\Gamma}^{H, n} \delta t \\
f_{c}^{H, n} \delta t
\end{array}\right)+\left(\begin{array}{c}
\left.Z_{l, \Gamma}^{n} u^{H, h, n-1}\right|_{\Gamma_{H}} \\
0 \\
0
\end{array}\right) .
\end{gathered}
$$

Proof. Elimination of $u_{l}^{H, n}$ from (18) gives (20).

We notice that (20) implies a discretization on the composite grid, while, for solving that system, we have only used uniform grids and uniform grid solvers.

\section{Numerical experiments}

In this section we present the results of a $2 D$ numerical experiment. We solve the following time-dependent convection-diffusion equation

$$
\frac{\partial u}{\partial t}+\nabla u=\nabla^{2} u+f
$$

in $\Omega=(0,2) \times(0,1)$. The initial condition, the boundary condition and the source term $f$ are chosen is such a way that the exact solution of the problem is

$$
u=3-\tanh (25(x-t)+5(y-1)) .
$$

The exact solution (22) has at each time a region of high activity that covers a small part of $\Omega$. The problem is solved by means of LDC with different values of $H, h, \Delta t$ and $\delta t$. The spatial discretization is performed using finite differences both globally and locally. The backward Euler scheme is used for time discretization both on the global and the local grid. The local region is chosen in such a way that at time level $t_{n}$

$$
\Omega_{l}=\left(\left(t_{n}-0.2, t_{n}+0.4\right) \times(0,1)\right) \cap \Omega .
$$

In our tests we perform only one LDC iteration per time step. As a comparison, we also solve problem (21) using a single uniform global grid with grid size $h_{\text {unif }}=h$ and time step $\delta t_{\text {unif }}=\delta t$. At time $t=0.6$ we measure the maximum error $\epsilon_{\max }$ of the numerical approximations with respect to the 
exact solution (22). Table 1 shows that LDC can achieve practically the same accuracy as the uniform grid solver. Of course LDC is computationally less expensive than the uniform grid solver since the fine grid spacing and the small time step are adopted only in a limited part of the domain.

Table 1. Results of the numerical experiment

\begin{tabular}{cccccc}
\hline \multicolumn{2}{c}{ Grid size } & \multicolumn{2}{c}{ Time step } & \multicolumn{2}{c}{$\epsilon_{\text {max }}$} \\
$H$ & $h=h_{\text {unif }}$ & $\Delta t$ & $\delta t=\delta t_{\text {unif }}$ & LDC & Unif. grid \\
\hline $1 / 10$ & $H / 3$ & $1.0 \cdot 10^{-1}$ & $\Delta t / 3$ & $4.36 \cdot 10^{-2}$ & $4.33 \cdot 10^{-2}$ \\
$1 / 10$ & $H / 5$ & $1.0 \cdot 10^{-1}$ & $\Delta t / 5$ & $1.21 \cdot 10^{-2}$ & $1.18 \cdot 10^{-2}$ \\
$1 / 20$ & $H / 3$ & $2.5 \cdot 10^{-2}$ & $\Delta t / 3$ & $9.50 \cdot 10^{-3}$ & $9.50 \cdot 10^{-3}$ \\
$1 / 20$ & $H / 5$ & $2.5 \cdot 10^{-2}$ & $\Delta t / 5$ & $3.02 \cdot 10^{-3}$ & $3.02 \cdot 10^{-3}$ \\
\hline
\end{tabular}

\section{References}

[AMTB03] Anthonissen, M.J.H., Mattheij, R M.M., ten Thije Boonkkamp, J.H.M.: Convergence analysis of the local defect correction method for diffusion equations. Numer. Math., 95, 401-425 (2003)

[Ant05] Anthonissen, M.J.H.: Local defect correction techniques applied to a combustion problem. In: Kornhuber, R., Hoppe, R., Périaux, J., Pironneau, O., Widlund, O., Xu, J. (ed) Domain Decomposition Methods in Science and Engineering. Lecture Notes in Computational Science and Engineering, pages 185-192. Springer, Berlin Heidelberg New York (2005)

[BS99] Bennett, B.A.V., Smooke, M.D.: Local rectangular refinement with application to nonreacting and reacting fluid flow problems. J. Comput. Phys., 151, 684-727 (1999)

[FR96] Ferket, P.J.J., Reusken, A.A.: Further analysis of the local defect correction method. Computing, 56, 117-139 (1996)

[Hac84] Hackbusch, W.: Local defect correction and domain decomposition techniques. In: Böhmer, K., Stetter, H.J. (ed) Defect Correction Methods. Theory and Applications. Computing, Suppl. 5, pages 89-113. Springer, Wien New York (1984)

[MAM04] Minero, R., Anthonissen, M.J.H., Mattheij, R.M.M.: A local defect correction technique for time-dependent problems. Technical Report CASA 04-25, Eindhoven University of Technology, Eindhoven (October 2004). To appear in Numer. Methods Partial Differential Equations 